\title{
Heat shock factor 2 is associated with the occurrence of lung cancer by enhancing the expression of heat shock proteins
}

\author{
YUN-HUA ZHONG ${ }^{1,2}$, HONG-ZHONG CHENG ${ }^{1,3}$, HAO PENG $^{1,3}$, SHI-CONG TANG $^{4}$ and PING WANG ${ }^{1,3}$ \\ ${ }^{1}$ Faculty of Life Science and Technology, Kunming University of Science and Technology, Kunming, Yunnan 650500; \\ ${ }^{2}$ Cadres Treatment Section; ${ }^{3}$ Thoracic Surgery, The First People's Hospital of Yunnan Province, Kunming, Yunnan 510032; \\ ${ }^{4}$ Cadres Health Care, The Affiliated Tumor Hospital of Guangxi Medical University, Nanning, Guangxi 530021, P.R. China
}

Received July 3, 2015; Accepted October 7, 2016

DOI: $10.3892 / 01.2016 .5368$

\begin{abstract}
Cancer is the leading cause of morbidity and mortality worldwide, particularly lung cancer. Heat shock proteins and their upstream heat shock factors are involved in the occurrence of cancer and have been widely researched. However, the role of heat shock factor 2 (HSF2) in lung cancer remains unclear. In the present study, expression levels of HSF2 in lung cancer tissues from 50 lung cancer patients were detected by reverse transcription quantitative polymerase chain reaction, and 76\% (38/50) were upregulated compared with the matched normal tissues. This suggested possible involvement of HSF2 in lung cancer. To additionally investigate the role of HSF2 in lung cancer occurrence, a plasmid encoding HSF2 was constructed. HSF2 was over expressed in normal lung epithelial BEAS-2B cells and lung cancer A549 cells. The results showed that HSF2 overexpression promoted cell proliferation and cell migration in BEAS-2B and A549 cells. Additional experiments showed that the HSF2-induced cell proliferation and cell migration were dependent on induction of HSPs, particularly HSP27 and HSP90, as co-transfection of HSP27 small interfering RNA (siRNA) or HSP90 siRNA attenuated HSF2-induced cell growth and migration. In conclusion, the present study showed that HSF2 is aberrantly expressed in lung cancer, and it may be an upstream regulator of HSPs, which may strongly affect cell growth and cell migration. Additional studies are required to explain the detailed mechanism between lung cancer, HSF2, HSPs and other possible signaling pathways.
\end{abstract}

Correspondence to: Dr Ping Wang, Thoracic Surgery, The First People's Hospital of Yunnan Province, 57 Jinbi Road, Kunming, Yunnan 510032, P.R. China

E-mail: wangping2005@hotmail.com

Key words: lung cancer, heat shock factor 2, heat shock protein 27, heat shock protein 90

\section{Introduction}

Cancer is the leading cause of morbidity and mortality worldwide, among which lung cancer accounts for more mortalities than other cancers (1). The occurrence of lung cancer is associated with complicated factors, including family history, infection (2), air pollution and tobacco use (3). Numerous genes are involved in the occurrence of lung cancer. Among them, numerous members of heat shock proteins (HSPs) are overexpressed in a wide range of human cancers and are implicated in tumor cell proliferation, differentiation and invasion, and also recognition by the immune system (4).

The heat shock responses were identified by Ritossa in 1962 in Drosophila in response to elevated temperatures (5). The heat shock responses are ubiquitous, existing in all organisms to protect cells against harmful conditions, including heat shock, oxidative stress or inflammation $(6,7)$. The synthesis of HSPs is the typical cellular response to stress. HSPs aid cells to facilitate refolding or degradation of misfolded and aggregated proteins induced by stress. HSPs are involved in numerous basic cell processes, including cell proliferation, cell differentiation and apoptosis. Cancer is characterized by an aberrant level of cell growth, cell differentiation and apoptosis. It was previously found that altered expression of HSPs has been reported in almost all classes of tumors. Increased levels of HSP27, relative to its level in non-transformed cells, have been detected in a number of cancers, such as breast cancer, endometrial cancer and leukemia $(6,8)$. Elevated expression of members of the HSP70 family has also been reported in high-grade malignant tumors (9). HSP90 family members, including Hsp90 $\alpha$ and Hsp90 $\beta$, are overexpressed in numerous types of cancers $(10,11)$.

A stress-responsive promoter element can be found upstream of the site of transcription initiation of HSP genes, and it is termed the heat shock element (HSE). Heat shock factors (HSFs) can bind to HSEs, and thus regulate the expression of HSPs (12). In total 3 HSFs (HSF1, HSF2 and HSF4) have been characterized in human cells (13). Among them, HSF1 has been associated with the occurrence of cancer (14). HSF4 is also associated with cancer, and inactivation of HSF4 induces cellular senescence and suppresses tumorigenesis in vivo (15). Thus, in the present study, the expression level of HSF2 in lung cancer was investigated and the cellular role 
of HSF2, including cell proliferation and cell migration, was characterized.

\section{Materials and methods}

Ethics. The present study was approved by the Medical Ethics Committee of Kunming University of Science and Technology (Kunming, China). Human samples were used in accordance with the requirements of Medical Ethics Committee of Kunming University of Science and Technology, under the guidelines of the World Medical Assembly (Declaration of Helsinki). Written informed consent was obtained from the patients' families.

Lung tissue samples. Lung specimens $(\mathrm{n}=50)$ were obtained from the tumor and an adjacent non-cancerous area $\geq 6 \mathrm{~cm}$ from the tumor tissues of 50 patients with lung cancer from Yunnan Province at the First People's Hospital of Yunnan Province (Kunming, China) between April 2014 and January 2015, as previously described $(16,17)$. The non-neoplastic tissue was confirmed to lack tumor cell infiltration using histological analysis. The tissues were immediately placed in liquid nitrogen and stored at $-80^{\circ} \mathrm{C}$ until use.

RNA extraction and polymerase chain reaction (PCR). RNA extraction and first-strand cDNA synthesis was assessed as previously described (18). For quantitative PCR (qPCR) of HSF2, the following primers were used: HSF2 forward, 5'-AAG TTCAGGCAGTGATGGCA-3' and reverse, 5'-TGCACAGAA CTAGTGAAAAGATCA-3'; glyceraldehyde 3-phospahte dehydrogenase forward, 5'-GAAGGTCGGAGTCAACGG AT-3' and reverse, 5'-GAGGGATCTCGCTCCTGGAAG-3'. qPCR was assessed using a continuous fluorescence detector (Opticon Monitor; Bio-Rad Laboratories Inc., Hercules, CA, USA) and PCR was assessed using an SYBR Green qPCR kit according to the manufacturer's protocol (Tiangen Bio, Inc., Beijing, China) with the following reaction conditions: Initial denaturation at $95^{\circ} \mathrm{C}$ for $1 \mathrm{~min}$ followed by 40 cycles at $95^{\circ} \mathrm{C}$ for $15 \mathrm{sec}, 60^{\circ} \mathrm{C}$ for $15 \mathrm{sec}$ and $72^{\circ} \mathrm{C}$ for $20 \mathrm{sec}$. Fluorescence curve analysis was assessed using Opticon Monitor software. The identities of qPCR products were confirmed by DNA sequencing. The relative gene expression in 2 different samples were compared using the $2^{-\Delta \Delta \mathrm{Cq}}$ method (19).

Western blot analysis. Western blot analysis of the tissues and the cells were performed as previously described $(16,20,21)$. Tissue and cell samples were homogenized in radioimmunoprecipitation assay buffer containing a protease inhibitor cocktail (Roche Applied Science, Penzburg, Germany). Samples (containing $50 \mu \mathrm{g}$ of protein) were loaded onto sodium dodecyl sulfate-polyacrylamide electrophoresis gel, electrophoresed and then electro-transferred onto a polyvinylidene fluoride membrane. The membrane was subsequently blocked with $3 \%$ bovine serum albumin and incubated with a rabbit anti-human HSF2 polyclonal antibody (dilution, 1:1,000; cat. no. SC-13056; Santa Cruz Biotechnology, Inc., Dallas, TX, USA) and a horseradish peroxidase-conjugated goat anti-rabbit IgG secondary antibody (dilution, 1:5,000; cat. no. SC-2004; Santa Cruz Biotechnology, Inc.). Protein bands were visualized using Super Signal reagents (Thermo Fisher

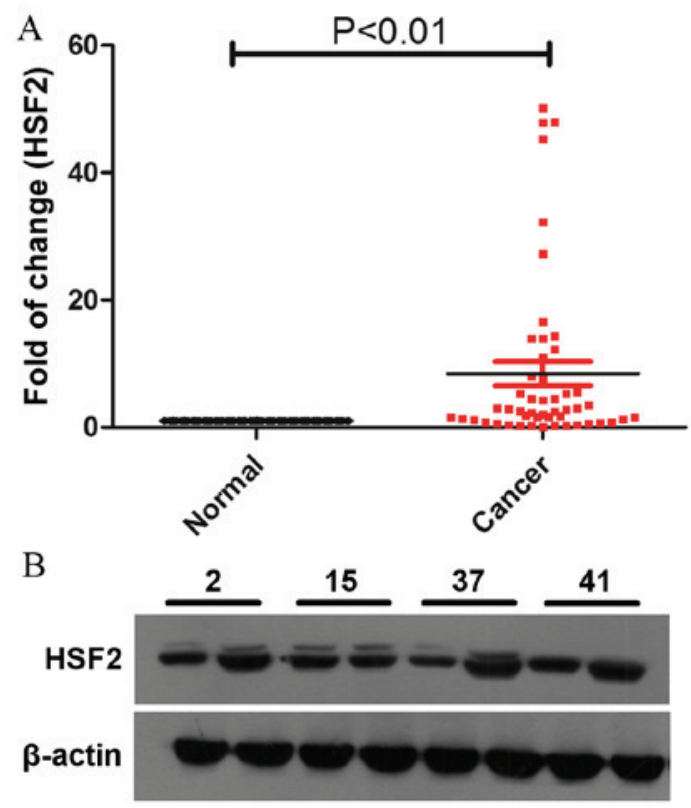

Figure 1. Level of HSF2 was significantly upregulated in lung cancer tissues. (A) HSF2 expressions in lung cancer tissue and matched normal tissues were detected in 50 lung adenocarcinoma patients from Yunnan, China by reverse transcription-quantitative polymerase chain reaction. The HSF2 mRNA level was increased by $76 \%(38 / 50)(\mathrm{P}=0.0003$, paired Wilcoxon test). (B) Matched normal and cancer tissues from representative patients were analyzed by western blotting $(n=4)$. The results showed increased protein levels in cancer tissues. The western blotting results are representative of two independent experiments. HSF2, heat shock factor 2 .

Scientific, Inc., Waltham, MA, USA). For detection of HSP27, HSP47, HSP70 and HSP90, the same methods were used. The antibodies were purchased from Santa Cruz Biotechnology, Inc and listed as follows: Rabbit anti-human Hsp27 polyclonal antibody (dilution, 1:1,000; cat. no. SC-9012); rabbit anti-human Hsp47 polyclonal antibody (dilution, 1:1,000; cat. no. SC-8352); rabbit anti-human Hsp70 polyclonal antibody (dilution, 1:1,000; cat. no. SC-25837); and rabbit anti-human Hsp90 polyclonal antibody (dilution, 1:1,000; cat. no. SC-7947).

HSF 2 overexpression plasmid construction. The cDNA of HSF2 was amplified from normal lung tissue using an RNA extraction kit (Tiangen Bio, Inc.) and the first strand cDNA sythesis kit (Takara Biotechnology Co., Ltd., Dalian, China). The PCR primers used were as follows: Forward, 5'-CGC GTTCGGGTGTAGAATTT-3'; and reverse, 5'-CATCCC ACCCCCGATCTTTC-3'. The cDNA with XhoI and BamHI restriction sites and flag tag at $\mathrm{N}$-terminal were amplified from the HSF2 cDNA with the following primers: Forward, 5'-AGATCTCGAGCCTGCGCCGCGTTAACAATGA-3'; and reverse, 5'-AGATGGATCCTTACTTATCGTCGTCATCCT TGTAATCTTAGCTATCAATAAGTGGCAT-3' (bold and underlined nucleotides are the restriction site of the enzymes). The PCR products and the plasmid pIRES2-EGFP were digested with XhoI and BamHI (Takara Biotechnology Co., Ltd.) and ligated with T4 ligase (Takara Biotechnology Co., Ltd.) and transformed into Escherichia coli DH5a competent cells (Tiangen Bio, Inc.). The positive clones were confirmed by sequencing, then the plasmid was amplified and purified for cell transfection. 
A

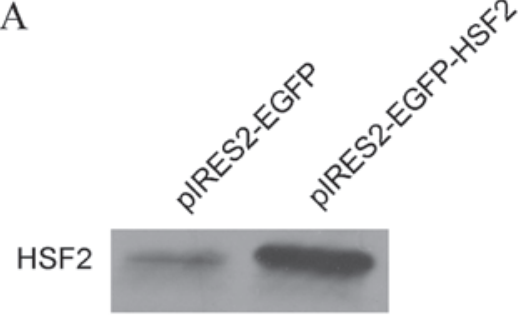

Flag
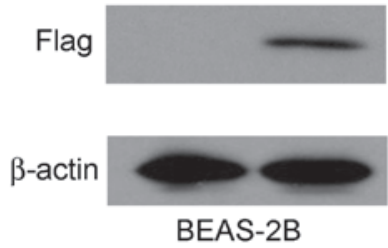

C

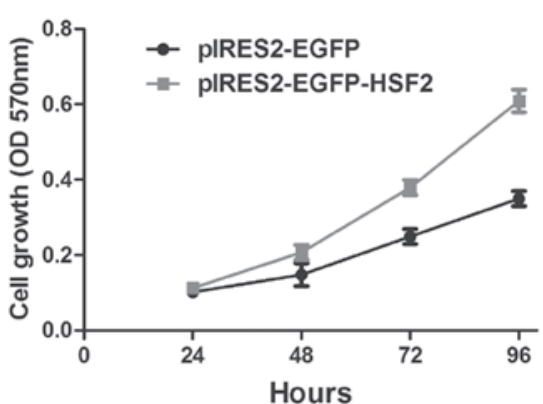

E

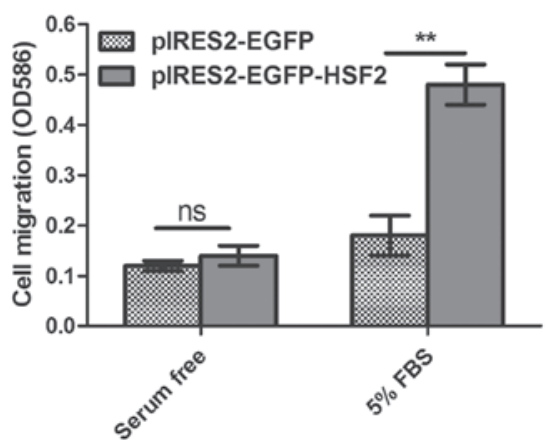

B

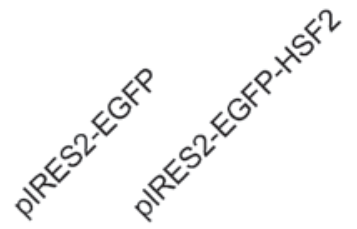

HSF2

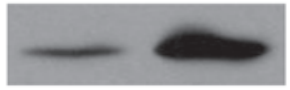

Flag

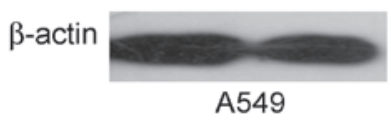

$\mathrm{D}$

A549

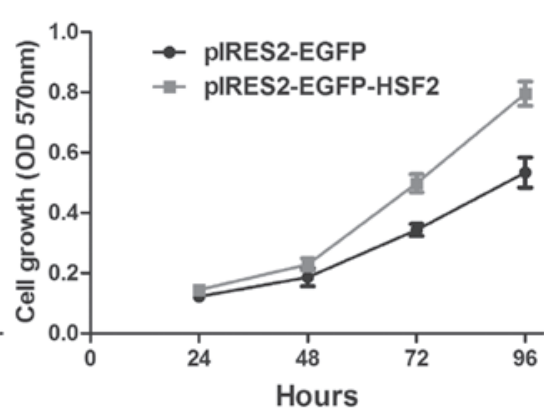

F

A549

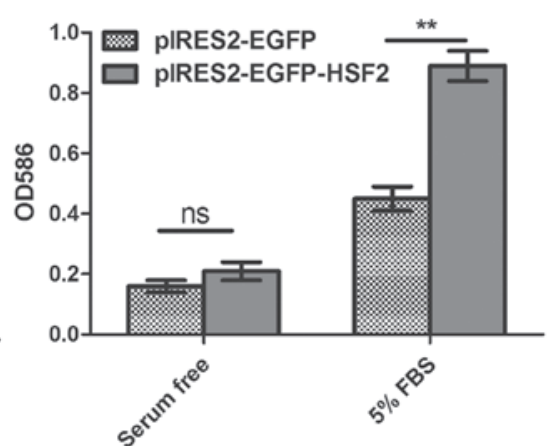

Figure 2. Overexpression of HSF2 promoted the growth migration of immortalized human bronchial BEAS-2B cells and lung cancer A549 cells. Western blot analysis confirmed the successful overexpression of Flag-tagged HSF2 in (A) BEAS-2B and (B) A549 cells. (C) HSF2 overexpression increased the cell proliferation of BEAS-2B cells by methyl thiazolyl tetrazolium assay. OD570 is associated with the number of cells. HSF2 overexpression promoted cell growth in BEAS-2B cells ( $\mathrm{P}=0.0006$ at 48 h). (D) HSF2 overexpression increased the cell proliferation of A549 cells by methyl thiazolyl tetrazolium assay. OD570 is associated with the number of cells. HSF2 overexpression promoted cell growth in A549 cells ( $\mathrm{P}=0.0021$ at 48 h). (E) HSF2 overexpression promoted the cell migration of BEAS-2B cells by Boyden chamber assay. OD586 is associated with the migrated cell number by staining with crystal violet. HSF2 overexpression promoted cell migration in BEAS-2B cells ( $\mathrm{P}=0.0027)$. (F) HSF2 overexpression promoted the cell migration of 549 cells by Boyden chamber assay. OD586 is associated with the migrated cell number by staining with crystal violet. HSF2 overexpression promoted cell migration in A549 cells $(\mathrm{P}=0.0041)$. The data were analyzed using Student's $t$-test for variance. Experimental values expressed as the mean \pm standard deviation. ${ }^{* *} \mathrm{P}<0.01 . \mathrm{HSF} 2$, heat shock factor 2; AD, optical density; FBS, fetal bovine serum.

Cell culture and transfection. The BEAS-2B and A549 cells were cultured in DMEM/F12 with $10 \%$ fetal bovine serum (FBS). The cells were grown in a humidified atmosphere containing $5 \% \mathrm{CO}_{2}$ at $37^{\circ} \mathrm{C}$. Transfection of cells was performed using Lipofectamine 2000 (Invitrogen; Thermo Fisher Scientific, Inc.) as previously described (22).

Cell proliferation assay. The cells were seeded onto a 96-well plate (1,000 cells/well) $24 \mathrm{~h}$ following transfection. Fresh medium supplemented with $10 \%$ FBS was added into the plates and changed every $24 \mathrm{~h}$. The number of viable cells was estimated by the methyl thiazolyl tetrazolium (MTT) method every $24 \mathrm{~h}$.

Cell migration assay. Cell migration was tested with a Boyden chamber assay, which utilized 24-well plates (Corning Incorporated, Corning, NY, USA) and Transwell plates (8 $\mu \mathrm{m}$ pore; EMD Millipore, Billerica, MA, USA). The bottom side of the Transwell plates were coated with collagen type 1 (10 $\mu \mathrm{g} / \mathrm{ml}$; Sigma-Aldrich; Merck Millipore, Darmstadt, Germany). Subsequent to being starved for $12 \mathrm{~h}$ with starvation medium (DMEM/F12) containing $0.25 \%$ bovine serum 


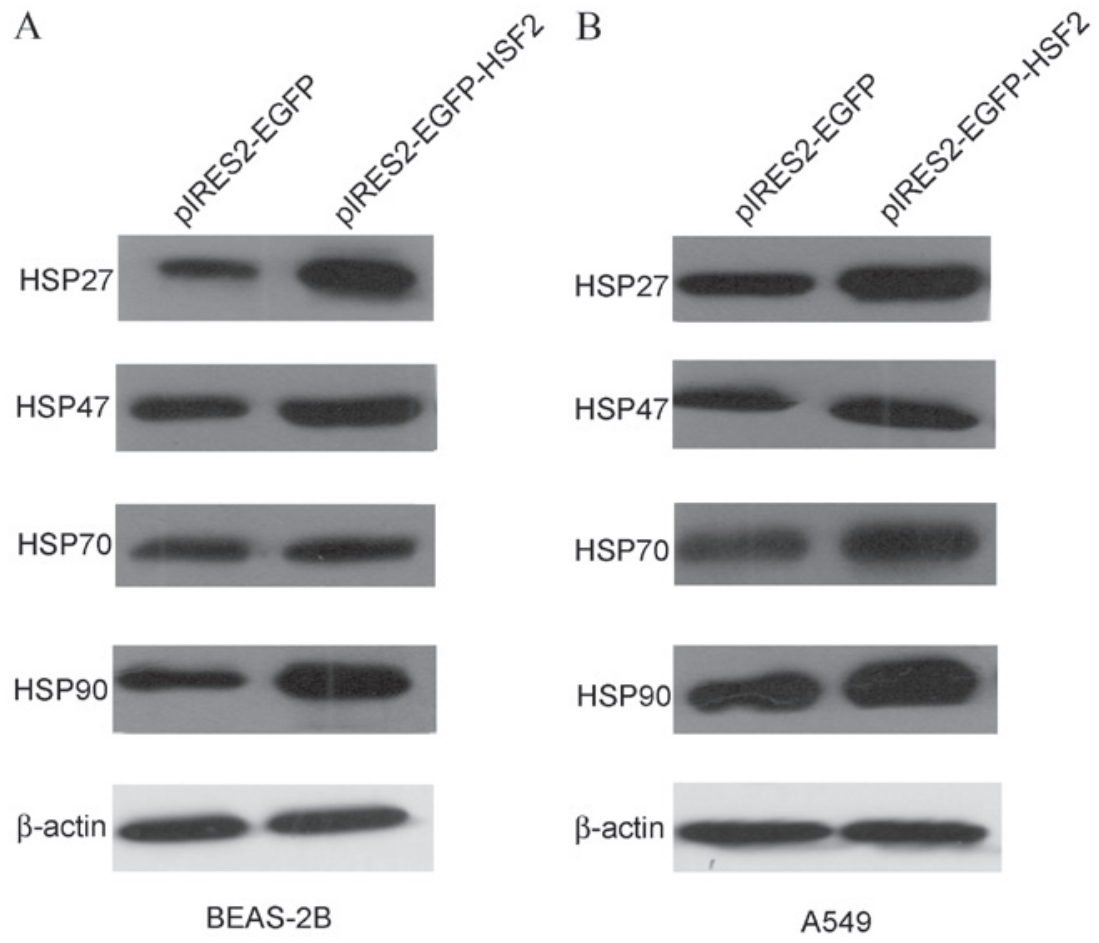

Figure 3. Overexpression HSF2 enhanced the expression of HSPs. The expression levels of HSP27, HSP47, HSP70 and HSP90 were detected by western blot analysis in (A) BEAS-2B cells and (B) A549 cells $48 \mathrm{~h}$ following transfection of pIRES2-EGFP control plasmid or plasmid encoding HSF2 (pIRES2-EGFP-HSF2). The western blotting results are representative of two independent experiments. HSF2, heat shock factor 2; HSP, heat shock protein.

albumin, the cells were detached with cell dissociation buffer, then $1 \times 10^{5}$ cells were planted into the top of each chamber. Medium containing 1\% FBS was added into the bottom of the chamber for $16 \mathrm{~h}$. Migrated cells were dyed with crystal violet. Bound crystal violet was eluted with $1 \mathrm{ml} 10 \%$ acetic acid and the migration activity was expressed as the value monitored at $586 \mathrm{~nm}$ of extraction.

Statistical analysis. Differences in the numerical data between the cancer and adjacent normal tissues were evaluated using the paired Wilcoxon test. The in vitro data were analyzed with Student's $t$-test for variance. Experimental values were expressed as the mean \pm standard deviation. A value of $\mathrm{P}<0.05$ was considered to indicate a statistically significant difference.

\section{Results}

HSF 2 is upregulated in tumor tissues in lung cancer patients. A total of 50 matched normal lung and lung cancer tissues were analyzed with RT-qPCR to detect the expression level of HSF2 (Fig. 1A). The results showed that 76\% (38/50) of the HSF2 expression levels in cancer tissues were upregulated compared with the normal lung tissues ( $\mathrm{P}=0.0003$; Fig. 1A). The protein levels of HSF2 were also detected by western blotting, and the results showed that the protein levels of HSF2 were upregulated, which is consistent with the results from RT-qPCR (Fig. 1B).

HSF 2 overexpression enhances the cell proliferation and cell migration in human lung epithelial cells and lung cancer cells. Since HSF2 is upregulated in lung cancer, it is reasonable to infer that it may affect the basic routine of lung epithelial cells and cancer cells, including cell proliferation and cell migration. Thus, a plasmid encoding HSF2 was constructed and transfected into human lung epithelia cell line BEAS-2B (Fig. 2A). The growth of HSF2-overexpressing BEAS-2B cells was monitored by MTT assay. The results showed that the HSF2 overexpression markedly enhanced the proliferation ability of BEAS-2B cells ( $\mathrm{P}=0.0006$; Fig. $2 \mathrm{C})$. The effect of HSF2 overexpression on the lung cancer A549 cell line was also investigated (Fig. 2B), and similar results were acquired $(\mathrm{P}=0.0021$; Fig. 2D). Apart from cell proliferation, enhanced cell migration was another characteristic of tumorigenesis. Thus, cell migration was tested using a Boyden chamber assay. The results showed that HSF2 overexpression significantly promoted the cell migration of BEAS-2B cells and A549 cells (Fig. 2E and F).

HSF 2 overexpression promotes the expression of HSPs. As the upstream regulator of HSP, HSF2 may regulate the expression level of HSP27, HSP47, HSP70 and HSP90. The present study additionally tested whether HSF2 overexpression can affect the expression of these HSPs. Subsequent to overexpression of HSF2 in BEAS-2B cells, the expression level of HSPs was semi-quantified by western blot analysis. All 4 HSPs, consisting of HSP27, HSP47, HSP70 and HSP90, were upregulated following HSF2 overexpression. Among them, HSP47 and HSP70 were only slightly upregulated, but the expression level of HSP27 and HSP90 were increased (Fig. 3A). However, HSF2 overexpression upregulated all 4 HSPs in A549 cells (Fig. 3B). These inconsistent results suggest that the regulation mechanism through which HSF2 affects the expression of HSPs may be different in normal epithelial cells and cancer cells. 

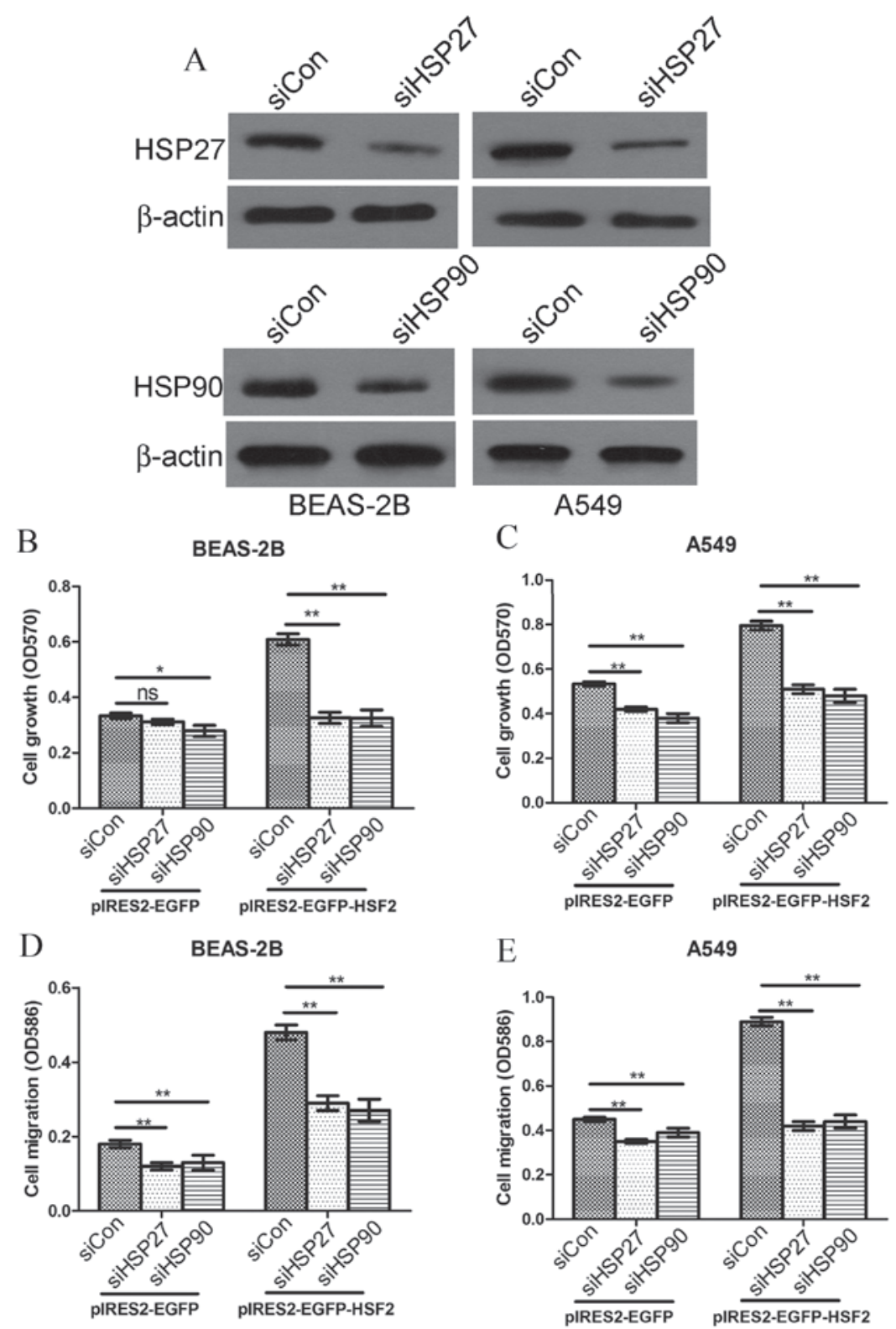

Figure 4. HSF2-promoted cell growth and cell migration is dependent on induction of upregulation of HSPs, particularly HSP27 and HSP90. (A) The representative western blot showed the protein level of knockdown degree of HSP27 and HSP90. (B) Co-transfection of HSP27 or HSP90 siRNA with pIRES2-EGFP-HSF2 significantly attenuated HSF2 promoted cell growth of BEAS-2B cells. (C) Co-transfection of HSP27 or HSP90 siRNA with pIRES2-EGFP-HSF2 significantly attenuated HSF2 promoted cell growth of A549 cells. (D) Co-transfection of HSP27 or HSP90 siRNA with pIRES2-EGFP-HSF2 significantly attenuated HSF2-promoted cell migration of BEAS-2B cells. (E) Co-transfection of HSP27 or HSP90 siRNA with pIRES2-EGFP-HSF2 significantly attenuated HSF2-promoted cell migration of A549 cells. The data were analyzed with Student's $t$-test for variance. Experimental values expressed as the mean \pm standard deviation. ${ }^{*} \mathrm{P}<0.01$; ${ }^{* *} \mathrm{P}<0.01$; Ns, not significant or $\mathrm{P}>0.05$; HSF2, heat shock factor 2; HSP, heat shock protein; siRNA, small interfering RNA; OD, optical density.

HSF 2 promoted cell proliferation and cell migration is dependent of its induced upregulation of HSP27 and HSP90. Since HSF2 overexpression can promote lung epithelial cell and lung cancer cell proliferation and migration, and at the same time upregulate HSP, particularly HSP27 and HSP90, it is reasonable to infer that HSP27 and HSP90 is pivotal for HSF2 functioning in promoting cell proliferation and cell migration. Thus, in the present study, HSP27 or HSP90 siRNA (or a combination of the two) were co-transfected with HSF2-overexpressing plasmids into BEAS-2B and A549 cells to assess the role of HSP27 and HSP90 in HSF2 function (Fig. 4A). The results showed that HSP27 and HSP90 RNA interference significantly attenu- ated HSF2 enhanced cell growth (Fig. 4B) and cell migration (Fig. 4D) on BEAS-2B cells. Similar results were obtained in A549 cells (Fig. 4C and E).

\section{Discussion}

Cancer is the leading cause of morbidity and mortality worldwide, and lung cancer accounts for a large proportion. Therefore, exploring the molecular and cellular mechanisms of lung cancer is important. Early screening strategies can be developed, and deciphering the mechanism underlying tumorigenesis may facilitate the development of new strat- 
egies to successfully treat cancer and cancer-associated diseases (23).

Increasing evidence suggests that changes in the cellular microenvironment contribute to tumorigenesis $(24,25)$. There are numerous bodily responses to microenvironmental changes, including inflammation. Among these responses, heat shock responses are the most conserved among all types of life, from bacterial to mammalian (26). Heat shock responses are generally accepted to be associated with tumor growth and metastasis (4). Numerous studies have focused on the role of HSPs on tumor growth and metastasis (27-29). As the upstream regulators of HSPs, HSFs may be also involved in cancer. Among the 3 members of HSFs, HSF1 has been well characterized for its association with cancer. A clinical study showed that high levels of HSF1 are associated with poor prognosis in breast cancer (30). It is also reported that HSF1 is involved in tumor progression via regulation of hypoxia-inducible factor 1 and RNA-binding protein HuR (31). However, the other two members, HSF2 and HSF4, have not been well characterized in cancer. Thus, in the present study, the expression level of HSF2 in lung cancer tissues was detected in 50 lung cancer patients. It was found that HSF2 level is upregulated in the majority of lung cancer tissues, suggesting that HSF2 may be involved in lung cancer growth and/or metastasis.

HSF1 is activated in response to the traditional heat shock stimuli. However, it is generally accepted that HSF2 is not activated in response to classical stress stimuli, but under developmentally associated conditions $(32,33)$. Coincidentally, with the recent progress in developmental biology and cancer biology, more and more similarities between early embryo development and tumorigenesis have been found (34). Thus, the upregulation of HSF2 in tumorigenesis complements current research. Therefore, in the next step, the biological effect of HSF2 on basic cellular effects was tested. The results showed that HSF2 promoted cellular proliferation and cellular migration in vitro, which may contribute to tumorigenesis in vivo.

The underlying mechanism of HSF2 promoting cell proliferation and cell migration may be complicated. A previous study showed that HSF2 binds to the HSP90, HSP27, HSP70 and c-Fos and modulates their expression (35). In the present study, it was assessed whether HSF2 affected the expression of these HSPs, and found that HSF2 overexpression indeed promoted the expression of HSP27, HSP47, HSP70 and HSP90, particularly HSP27 and HSP90. This may partially explain why HSF2 promotes cell proliferation and cell migration. The knockdown of HSP27 or HSP90 can significantly attenuate the HSF2 induced cell proliferation and cell migration. However, additional research is required to understand the role of HSF2 in cancer. The involvement of certain signaling pathways, including the MAP kinase pathway, which utilizes ERK1/2, P38 and JNK in HSF2-regulated cell proliferation and cell motility, may be investigated in additional studies. There are a limited number of studies about the HSF2 expression and human diseases. A previous study showed that HSF2 is upregulated in inflammatory bowel diseases by enhancing the production of inflammatory cytokines. Inflammation, particularly chronic inflammation, is closely associated with the occurrence of cancer. Whether it is the same or a similar condition in lung cancer requires investigation. Additional detailed studies are required to answer whether the upregulation of HSF is the cause or the result of lung cancer, which is important for understanding the association between HSF2 upregulation and lung cancer occurrence.

In conclusion, to the best of our knowledge, the present study showed for the first time that HSF2 is upregulated in lung cancer and may be involved in tumorigenesis. HSF2 asserts its biological function by upregulating the expression of HSP and consequently affecting cell proliferation and cell migration. Whether HSF2 acts alone or with other proteins may require additional investigation. In addition, whether HSF2 is a good diagnostic marker or drug target requires additional research.

\section{Acknowledgements}

This study was supported by Yunnan Department of Natural Science and Kunming Medical University (grant no., 2013FB197) and The First People's Hospital of Yunnan Province Internal Institutions (grant no., 2016NS204).

\section{References}

1. Siegel R, Naishadham D and Jemal A: Cancer statistics, 2013, CA Cancer J Clin 63: 11-30, 2013.

2. Zhan P, Suo LJ, Qian Q, Shen XK, Qiu LX, Yu LK and Song Y: Chlamydia pneumoniae infection and lung cancer risk: A meta-analysis. Eur J Cancer 47: 742-747, 2011.

3. Hecht SS: Tobacco smoke carcinogens and lung cancer. J Natl Cancer Inst 91: 1194-1210, 1999.

4. Ciocca DR and Calderwood SK: Heat shock proteins in cancer: Diagnostic, prognostic, predictive, and treatment implications. Cell Stress Chaperones 10: 86-103, 2005.

5. Ritossa F: A new puffing pattern induced by temperature shock and DNP in Drosophila. Experientia 18: 571-573, 1962.

6. Jolly C and Morimoto RI: Role of the heat shock response and molecular chaperones in oncogenesis and cell death. J Natl Cancer Inst 92: 1564-1572, 2000.

7. Morimoto RI: Cells in stress: Transcriptional activation of heat shock genes. Science 259: 1409-1410, 1993.

8. Ciocca DR, Oesterreich S, Chamness GC, McGuire WL and Fuqua SA: Biological and clinical implications of heat shock protein 27,000 (Hsp27): A review. J Natl Cancer Inst 85: $1558-1570,1993$

9. Ralhan R and Kaur J: Differential expression of Mr 70,000 heat shock protein in normal, premalignant, and malignant human uterine cervix. Clin Cancer Res 1: 1217-1222, 1995.

10. Jameel A, Skilton RA, Campbell TA, Chander SK, Coombes RC and Luqmani YA: Clinical and biological significance of HSP89 alpha in human breast cancer. Int J Cancer 50: 409-415, 1992.

11. Yufu Y, Nishimura J and Nawata H: High constitutive expression of heat shock protein 90 alpha in human acute leukemia cells. Leuk Res 16: 597-605, 1992.

12. Wu C: Heat shock transcription factors: Structure and regulation, Annu Rev Cell Dev Biol 11: 441-469, 1995.

13. Morimoto RI: Regulation of the heat shock transcriptional response: Cross talk between a family of heat shock factors, molecular chaperones and negative regulators. Genes Dev 12: 3788-3796, 1998.

14. Engerud H, Tangen IL, Berg A, Kusonmano K, Halle MK, Oyan AM, Kalland KH, Stefansson I, Trovik J, Salvesen HB and Krakstad C: High level of HSF1 associates with aggressive endometrial carcinoma and suggests potential for HSP90 inhibitors. Br J Cancer 111: 78-84, 2014.

15. Jin X, Eroglu B, Cho W, Yamaguchi Y, Moskophidis D and Mivechi NF: Inactivation of heat shock factor Hsf4 induces cellular senescence and suppresses tumorigenesis in vivo. Mol Cancer Res 10: 523-534, 2012.

16. Jiang P, Yu GY, Zhang Y, Xiang Y, Hua HR, Bian L, Wang CY, Lee WH and Zhang Y: Down-regulation of protease-activated receptor 4 in lung adenocarcinoma is associated with a more aggressive phenotype. Asian Pac J Cancer Prev 14: 3793-3798, 2013. 
17. Yu G, Jiang P, Xiang Y, Zhang Y,Zhu Z, Zhang C, Lee S, Lee W and Zhang Y: Increased expression of protease-activated receptor 4 and trefoil factor 2 in human colorectal cancer. PLoS One 10: e122678, 2015.

18. Xiang Y, Gao Q, Su W, Zeng L, Wang J, Hu Y, Nie W, Ma X, Zhang Y, Lee W and Zhang Y: Establishment, characterization and immortalization of a fibroblast cell line from the Chinese red belly toad Bombina maxima skin. Cytotechnology 64: 95-105, 2012.

19. Livak KJ and Schmittgen TD: Analysis of relative gene expression data using real-time quantitative PCR and the 2(-Delta Delta C(T)) Method. Methods 25: 402-408, 2001.

20. Xiang Y, Wang X, Yan C, Gao Q, Li SA, Liu J, Zhou K, Guo X, Lee W and Zhang Y: Adenosine-5'-triphosphate (ATP) protects mice against bacterial infection by activation of the NLRP3 inflammasome. PLoS One 8: e63759, 2013.

21. Wang YJ, Guo XL, Li SA, Zhao YQ, Liu ZC, Lee WH, Xiang Y and Zhang Y: Prohibitin is involved in the activated internalization and degradation of protease-activated receptor 1. Biochim Biophys Acta 1843: 1393-1401, 2014.

22. Huang F, Lin C, Shi YH and Kuerban G: MicroRNA-101 inhibits cell proliferation, invasion and promotes apoptosis by regulating cyclooxygenase-2 in Hela cervical carcinoma cells. Asian Pac J Cancer Prev 14: 5915-5920, 2013.

23. Lei YM, Zu YF, Wang J, Bai S, Shi YF, Shi R, Duan J, Cui D, Chen J, Xiang Y and Dong J: Interleukin-1 $\beta$-mediated suppression of microRNA-101 and upregulation of enhancer of zeste homolog 2 is involved in particle-induced lung cancer. Med Oncol 32: 387, 2015.

24. Hu M, Yao J, Cai L, Bachman KE, van den Brûle F, Velculescu V and Polyak K: Distinct epigenetic changes in the stromal cells of breast cancers. Nat Genet 37: 899-905, 2005.

25. Gao F, Liang B, Reddy ST, Farias-Eisner R and Su X: Role of inflammation-associated microenvironment in tumorigenesis and metastasis. Curr Cancer Drug Targets 14: 30-45, 2014.
26. Fulda S, Gorman AM, Hori O and Samali A: Cellular stress responses: Cell survival and cell death. Int J Cell Biol 2010: 214074, 2010.

27. Tan JS, Ong Kc KC and Rhodes A: The role of heat shock proteins and glucose regulated proteins in cancer. Malays J Pathol 38: 75-82, 2016.

28. Conroy SE and Latchman DS: Do heat shock proteins have a role in breast cancer? Br J Cancer 74: 717-721, 1996.

29. Lianos GD, Alexiou GA, Mangano A, Mangano A, Rausei S, Boni L, Dionigi G and Roukos DH: The role of heat shock proteins in cancer. Cancer Lett 360: 114-118, 2015.

30. Santagata S, Hu R, Lin NU, Mendillo ML, Collins LC, Hankinson SE, Schnitt SJ, Whitesell L, Tamimi RM, Lindquist $S$ and Ince TA: High levels of nuclear heat-shock factor 1 (HSF1) are associated with poor prognosis in breast cancer. Proc Natl Acad Sci USA 108: 18378-18383, 2011.

31. Gabai VL, Meng L, Kim G, Mills TA, Benjamin IJ and Sherman MY: Heat shock transcription factor Hsf1 is involved in tumor progression via regulation of hypoxia-inducible factor 1 and RNA-binding protein HuR. Mol Cell Biol 32: 929-940, 2012

32. Loones MT, Rallu M, Mezger V and Morange M: HSP gene expression and HSF2 in mouse development. Cell Mol Life Sci 53: 179-190, 1997.

33. Pirkkala L, Nykänen P and Sistonen L: Roles of the heat shock transcription factors in regulation of the heat shock response and beyond. FASEB J 15: 1118-1131, 2001.

34. Ma Y, Zhang P, Wang F, Yang J, Yang Z and Qin H: The relationship between early embryo development and tumourigenesis. J Cell Mol Med 14: 2697-2701, 2010.

35. Wilkerson DC, Skaggs HS and Sarge KD: HSF2 binds to the Hsp90, Hsp27 and c-Fos promoters constitutively and modulates their expression. Cell Stress Chaperones 12: 283-290, 2007. 